\title{
BMJ Open Quality Putting veterans with heart failure FIRST improves follow-up and reduces readmissions
}

\author{
Serena Michelle Ogunwole, ${ }^{1}$ Jason Phillips, ${ }^{2}$ Amber Gossett, ${ }^{3}$ \\ John Richard Downs ${ }^{4}$
}

To cite: Ogunwole SM, Phillips J, Gossett A, et al. Putting veterans with heart failure FIRST improves follow-up and reduces readmissions. BMJ Open Quality 2019;8:e000386. doi:10.1136/ bmjoq-2018-000386

Received 23 March 2018 Revised 1 December 2018 Accepted 3 December 2018

Check for updates

(c) Author(s) (or their employer(s)) 2019. Re-use permitted under CC BY-NC. No commercial re-use. See rights and permissions. Published by BMJ.

${ }^{1}$ Internal Medicine, University of Texas Health Science Center, San Antonio, Texas, USA

${ }^{2}$ Department of Cardiology, South Texas Veterans Health Care System, University of Texas Health Science Center, San Antonio, Texas, USA

${ }^{3}$ Clinical Pharmacology, South

Texas Veterans Health Care

System, San Antonio, Texas, USA

${ }^{4}$ Hospital Medicine Section,

Department of Medicine, South

Texas Veterans Health Care

System, University of Texas

Health Science Center, San

Antonio, Texas, USA

Correspondence to

Dr Serena Michelle Ogunwole;

michelle.ogunwole@gmail.com

\section{ABSTRACT}

Background Despite improvements in length of stay and mortality, congestive heart failure (CHF) remains the most common cause of 30-day readmissions to the hospital. Though multiple studies have found that early follow-up after discharge (eg, within 7 days) is critical to improving 30-day readmissions, implementation strategies are challenging in resource-limited settings. Here we present a quality improvement initiative aimed at improving early follow-up while maximising available resources.

Methods This was a medical resident-driven initiative. A process map of the discharge and follow-up appointment process was created that identified multiple areas for improvement. Based on these findings, a two-part intervention was implemented. First, heart failure discharge education with focus on early follow-up was disseminated to providers throughout the internal medicine department. Subsequently, improved identification of highrisk patients (Failure Intervention Risk StratificationTool) and innovative use of the existing electronic medical record (EMR) were employed to sustain and improve on gains from the first set of interventions.

Results We increased our 7-day follow-up rate from $47 \%$ to $57 \%(p=0.429)$ and decreased the average time to follow-up from 17.6 days to 8.7 days ( $p=0.016$ ) following the first intervention. The percentage of patients readmitted within 30 days after discharge at baseline (2012-2013) and following the first intervention (education and standardisation of follow-up scheduling) and second intervention (risk stratification, intensive follow-up and EMR change) was $25 \%$ and $21 \%$, respectively. Thirty-day mortality rate decreased from $10 \%$ in 2011 to $7.16 \%$ in December 2015.

Conclusion Close hospital discharge follow-up and identification of high-risk patients with CHF are useful approaches to reduce readmissions. Using the existing EMR tool for identifying high-risk patients and improving adherence to best practices is an effective intervention. In patients with CHF these strategies improved time to follow-up and 30-day readmissions while decreasing mortality.

\section{INTRODUCTION}

\section{Available knowledge}

Over a million patients are hospitalised in the USA for congestive heart failure (CHF) each year. ${ }^{12}$ As many as $25 \%$ of these patients are readmitted within 30 days of discharge, ${ }^{1}$ making CHF exacerbations, also known as acute decompensated heart failure (ADHF), the most common cause of 30-day hospital readmissions. From a financial standpoint, treatment of CHF is extremely costly; with direct and indirect costs totalling more than $\$ 37$ billion and projected to increase to nearly $\$ 70$ billion by the year $2030 .^{3}$ Additionally, the shift towards 30-day readmission rates as a quality measure has led to increased financial penalties for institutions with the highest readmission rates. ${ }^{4}$ With the prevalence of CHF increasing in the population, ${ }^{3}$ strategies to improve readmission rates for $\mathrm{ADHF}$ may be the most effective way to reduce cost and improve quality of care. ${ }^{2}$

\section{Local problem description}

Heart failure readmission rates at the South Texas Veterans Health Care System hospital were consistently higher than the national average. The risk-adjusted 30-day all-cause readmission rate for $\mathrm{ADHF}$ at the start of this project in fiscal year 2012-2013 was $25 \%$ (compared with $23 \%$ nationally $)^{5}$ with approximately $10 \%$ 30-day mortality. Efforts had been made locally to address education of patients in the hospital and follow-up appointment optimisation with marginal success.

\section{Rationale}

Several studies of patients hospitalised with ADHF have linked the reduction of 30-day readmission rates with early (within 7 days) physician follow-up after discharge. ${ }^{6}$ We describe an intervention aimed at ensuring all patients with a diagnosis of ADHF received early follow-up after discharge.

In addition to early follow-up, there is evidence to suggest that cardiology consultation may be useful for improving adherence to recommended guidelines for patients with heart failure. ${ }^{8}$ However, it is also noted that cardiology consultation may be costlier and have less available access than primary care. 
With this knowledge, we set out to create a standardised process to identify 'high-risk' patients who may benefit more from early cardiologist follow-up at the time of discharge.

\section{Aims}

The primary aim of this project was to increase the number of completed 7-day follow-up appointments from $47 \%$ to $100 \%$ for all patients discharged from the internal medicine service with the diagnosis of ADHF. In accordance with prior literature on the effect of early follow-up on readmissions, our secondary aim was to reduce the 30-day readmission rate for patients with ADHF to $20 \%$ at our hospital.

\section{METHODS \\ Context}

The South Texas Veterans Health Care System is a 493-bed tertiary care centre and has an academic affiliation with University of Texas Health San Antonio, serving as one of the primary teaching sites for Graduate Medical Education. Internal medicine residents rotating through the medicine wards care for veterans with a multitude of health issues, ADHF being one of the most common.

Internal medicine resident involvement in this project was driven in part by the training requirements of the Accreditation Council for Graduate Medical Education that requires all residents to participate in quality improvement $(\mathrm{QI})$ and patient safety activities. To meet these requirements, the residents participate in a longitudinal QI and patient safety curriculum that includes participation in the development of a project. Residents are divided into cohorts of 20-25 individuals led by a faculty mentor. Projects are chosen based on areas of interest highlighted by hospital leadership, or suggestions from within the resident cohort itself.

At an administration level, reducing the volume of heart failure readmissions had been identified as a local institutional priority. Accordingly, a team of pharmacists known as the Patient Aligned Care Team (PACT) began collecting data on postdischarge appointments, attendance rates of appointments and readmission rates for patients with ADHF. Additionally, a Heart Failure Workgroup, led by members of the PACT, was convened and met monthly to discuss the current state of heart failure readmissions and methods to improve rates. Institutional processes were put in place to help with improvements in coding, education, telehealth enrolment and access to clinic visits with some success; but there remained a need to champion further interventions at the provider level.

Prior to the start of this project, internal medicine residents were not involved in Heart Failure Workgroup meetings, and the internal medicine ward teams operated independently in scheduling follow-up for patients with ADHF.

In 2014, the internal medicine chief resident of quality and safety (Chief Resident of Quality and Safety is a 1-year postresidency position supported by the Veterans Affairs office. The position is part of a national programme aimed at increasing the number of faculty adept at teaching QI and patient safety topics) was asked to lead a cohort of residents in focusing on this issue. The team was asked to analyse the processes an internal medicine resident ward team would go through to admit, care for, discharge and follow-up patients with ADHF. In the early stages of evaluation, the discharge and follow-up processes quickly stood out as the areas with the most heterogeneity and potential to most impact readmissions.

\section{Process evaluation}

Residents directly involved in this project were encouraged to meet with personnel who were involved in the discharge and follow-up of patients from the hospital. During this process evaluation, they met with other residents on the wards who were placing discharge and follow-up orders, hospital floor clerks who received those orders, schedulers who made the follow-up appointments, outpatient physicians who follow-up the patients, and the patients themselves. Policies regarding service agreements between primary care clinics and inpatient hospital teams were also reviewed. A process map (figure 3) (figure 1) of the series of steps from discharge to follow-up was then developed.

The process map revealed several problems in the discharge process. First, the decision on time to follow-up appointment was not standardised. The physician team member who would determine the necessary time to follow-up (resident, fellow or attending) varied among different teams and the decision made was not evidence based but rather an estimation (eg, 1-2 weeks after discharge) based on time to follow-up for other common discharge diagnoses. Second, whether the patient saw a primary care provider (PCP) or a cardiologist at follow-up also varied widely and was determined by availability of appointments rather than medical necessity.

One of the most surprising discoveries of mapping out the process was the myriad of strategies residents used to obtain follow-up. These included direct calls to clinics, placing 'clerk orders' for a floor clerk to call and make the appointment, and placing consult orders to various clinics. In addition, it was noted that cardiology fellows would at times arrange appointments independently. These methods only worked during normal business hours. If a patient was discharged during the night or on a weekend the process became much less clear.

To validate the findings of the process map, a SurveyMonkey questionnaire was administered via email to all internal medicine residents asking what process they were using when discharging patients. Of 95 residents surveyed, 37 responded. Results of the survey were consistent with the process map: a wide variation among residents regarding how to make follow-up appointments was evident. The survey showed that most residents preferred to use the electronic medical record (EMR) as a communication tool to facilitate follow-up scheduling 


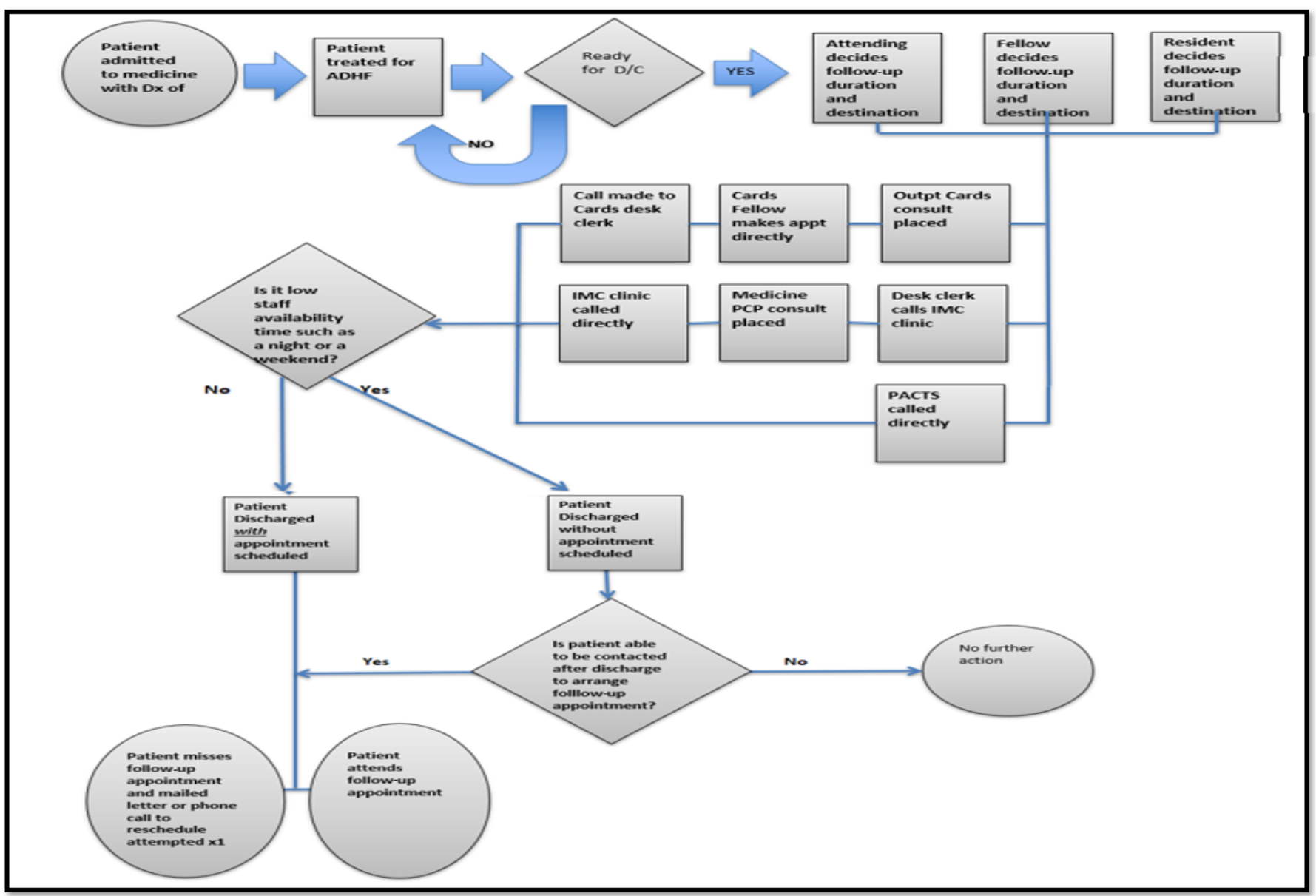

FIGURE 1. Process map of discharge work flow before intervention

Dx: Diagnoses, D/C: Discharge, IMC: Internal Medicine Clinic, ADHF: Acute decompensated heart failure, PACTS: Patient aligned care team specialist, PCP: Primary care provider

Figure 1 Process map of discharge work flow before intervention.

(67\% used the EMR to place a clerk request to schedule follow-up while $29 \%$ placed a consult to a PCR to request follow-up). A minority (22\%) relied on direct communication via phone call to a provider or clinic that would presumably take over the patient's care. Several conversations during resident cohort meetings revealed no standard education had been deployed to the residents about evidence-based recommendations on time to follow-up or best practices to arrange follow-up within their institutional environment.

Finally, a cause and effect diagram (figure 2) corroborated all the aforementioned evidence, and also revealed multiple system issues including a lack of contingency plan for follow-up scheduling during nights and weekends, limited appointment availability and institutional polices that made scheduling in subspecialty clinics difficult.

\section{INTERVENTIONS}

After multiple rounds of discussion with outpatient clinics, clerks and inpatient and outpatient schedulers, an approach to standardise the workflow process for follow-up appointments was created. Additionally, the PACT pharmacists offered to assist in seeing patients with
CHF recently discharged from the hospital. This dramatically increased the follow-up capacity and ability to see patients on short notice.

The first set of interventions involved education regarding the new process. A one-page flyer was placed in each medicine ward team room. The flyer included a step-by-step guide for the discharge process. The flyer was complete with a stepwise approach to arranging follow-up including numbers to dial (with backup numbers if no answer), and contingency plans for the nights and weekends. As residents rotate on and off the ward services frequently, the chief resident of quality and safety stopped by the team rooms on a weekly basis to orient new residents to the process.

With implementation of the educational flyer, time from discharge to outpatient follow-up was reduced by over 8 days (see the Results section for details). While these gains were promising, there was a growing concern about the sustainability of having one individual be responsible for reorientation and re-education as residents transitioned on and off service. With these potential challenges in mind, a stronger intervention was sought.

After reviewing evidence suggesting that follow-up with cardiology may improve adherence to recommended 


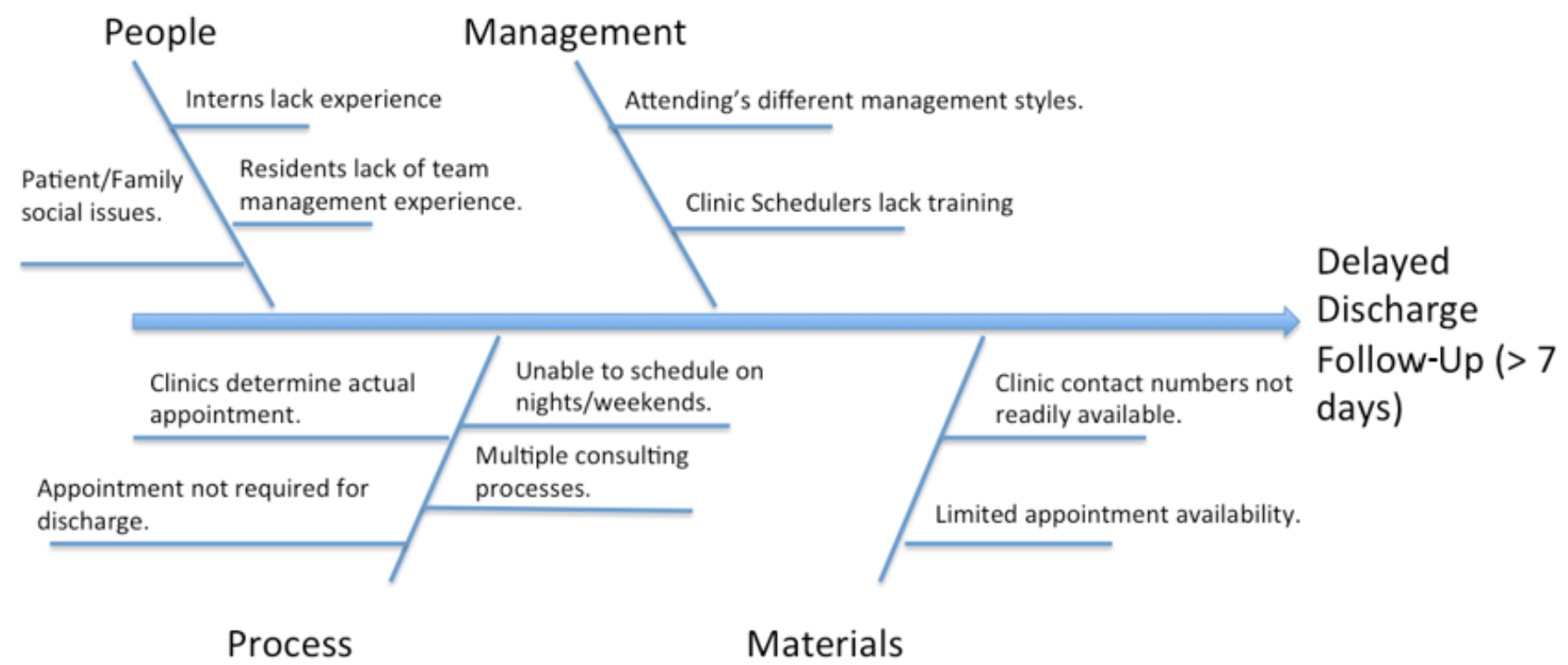

Figure 2 Cause and effect diagram for the problem of delayed discharge.

guidelines for patients with heart failure, ${ }^{8}$ we met with our local heart failure specialist, and discussed the need for specialist care in this complex population. However, we remained realistic in our expectations of a single specialist provider being able to manage an entire hospital system's CHF population. As a solution, our team sought to identify patients who would potentially derive the most benefit from early specialty follow-up with cardiology.

Through literature review and consultation with our heart failure specialist, we determined criteria to identify patients with heart failure at highest risk for readmission. These criteria included any one of the following:

1. Greater than two hospitalisations for ADHF in the past year.

2. Left ventricular ejection fraction less than $25 \%$.

3. New York heart failure class IV on admission.

4. Patients admitted for ADHF who had developed acute kidney injury (defined as greater $30 \%$ increase in creatinine from baseline) at the time of discharge.

The next set of interventions included modification of an existing protocol for intense follow-up (defined as providing weekly appointments, 1 month after discharge) for patients with ADHF with a provider capable of adjusting medications. Additionally, early cardiology follow-up was put in place for patients we predefined as 'high-risk' for readmission. Once these patients were identified, they were given follow-up with a cardiologist in the first week following discharge and continued weekly follow-up in the first month following discharge with their PCP or PACT pharmacist. Lastly, we took into consideration the resident survey results showing a preference for EMR orders and created an automatic discharge order set to help standardise the discharge and risk stratification process.

\section{Explanation of intervention}

In an effort to create an intervention that was a natural part of resident work flow, we looked to see what we could do to enhance the current discharge process. Since all patients already required an EMR-generated medication reconciliation prior to discharge, we decided to add a checkbox to the current medication reconciliation form. Whenever a resident was discharging a patient with a primary diagnosis of ADHF, they would select the checkbox, and a mandatory order set would populate. The order set contained the criteria that enabled residents to quickly risk stratify patients at high risk for ADHF readmission. As described previously, high-risk patients would have follow-up with the heart failure specialist in the first week after discharge, and then PACT pharmacists each week for the next 3 weeks. Patients who did not meet high-risk categorisation by the criteria would have follow-up with PACT pharmacists during weeks 1, 2 and 4, and with cardiology on week 3 . Regardless of who the patients would follow-up with, all first postdischarge appointments were scheduled within 7 days of discharge. Of note, patients discharged to hospice or a skilled nursing facility were excluded from this process.

On completion of the risk stratification portion, an autopopulated clerk order to make an appointment with the appropriate clinic was generated. These combined processes in the EMR helped streamline and standardise the process for follow-up appointments for patients admitted with ADHF.

\section{STUDY OF THE INTERVENTIONS}

\section{Data collection}

Data on baseline time to follow-up and follow-up completion rates were assessed via a retrospective chart review of patients with heart failure identified by primary admission diagnosis 
(International Classification of Diseases code) during the 1 November to 30 December 2014 period ( $\mathrm{n}=26$ ).

For local readmission rates, the Heart Failure Workgroup (working independently of the resident QI cohort) collected and analysed retrospective data as part of programmatic evaluation. They reviewed data between May 2012 and December 2015. Patient data were extracted from a VA Regional Data Warehouse for analysis. Patients were considered readmitted if the next admission date for any cause was within 30 days of the previous postdischarge date.

\section{Measures}

The primary outcome measure was the percentage of follow-up appointments completed within 7 days. In addition, we tracked the number of days until follow-up appointment as a process measure related to the primary outcome measure. Secondary outcome measures included 30-day readmission rates and 30-day mortality rate. Reduction in 30-day mortality rate was not an aim of this project, however 30-day mortality was evaluated as part of the programmatic evaluation, and thus were included in this study.

\section{ANALYSIS}

\section{Completed follow-up rate}

Data from 66 patient discharges were evaluated via retrospective chart review and taken as the percentage of patients who showed up to scheduled appointments within 7 days of discharge. A test of differences in proportions was carried out to evaluate significant differences in the rate of follow-up before and after the intervention.

Time to follow-up

Data from 66 patient discharges were plotted on an XMr control chart (figure 3) using an Excel template and the software package QI MACROS. Average time to follow-up at baseline and after the intervention was assessed via the $\mathrm{XMr}$ control chart. A two-sample t-test with unequal variance was used to compare the time to follow-up at baseline and following the first intervention.

\section{Local readmission rates}

A total of 204 all-cause 30-day readmissions occurred during the study period.

Local 30-day all-cause readmission rate data were obtained from the Veteran Administration Inpatient Evaluation Center (IPEC) report. (IPEC evaluates the 30-day unadjusted readmission rate of $\mathrm{CHF}$ as the proportion of patients with CHF who were readmitted for any cause to an acute ward within 30 days from the previous discharge date.)

Local risk-adjusted 30 -day mortality rates

These data were obtained through the Veterans Affairs Strategic Analytics for Improvement and Learning (SAIL) data set. (SAIL assesses 25 quality measures in areas such

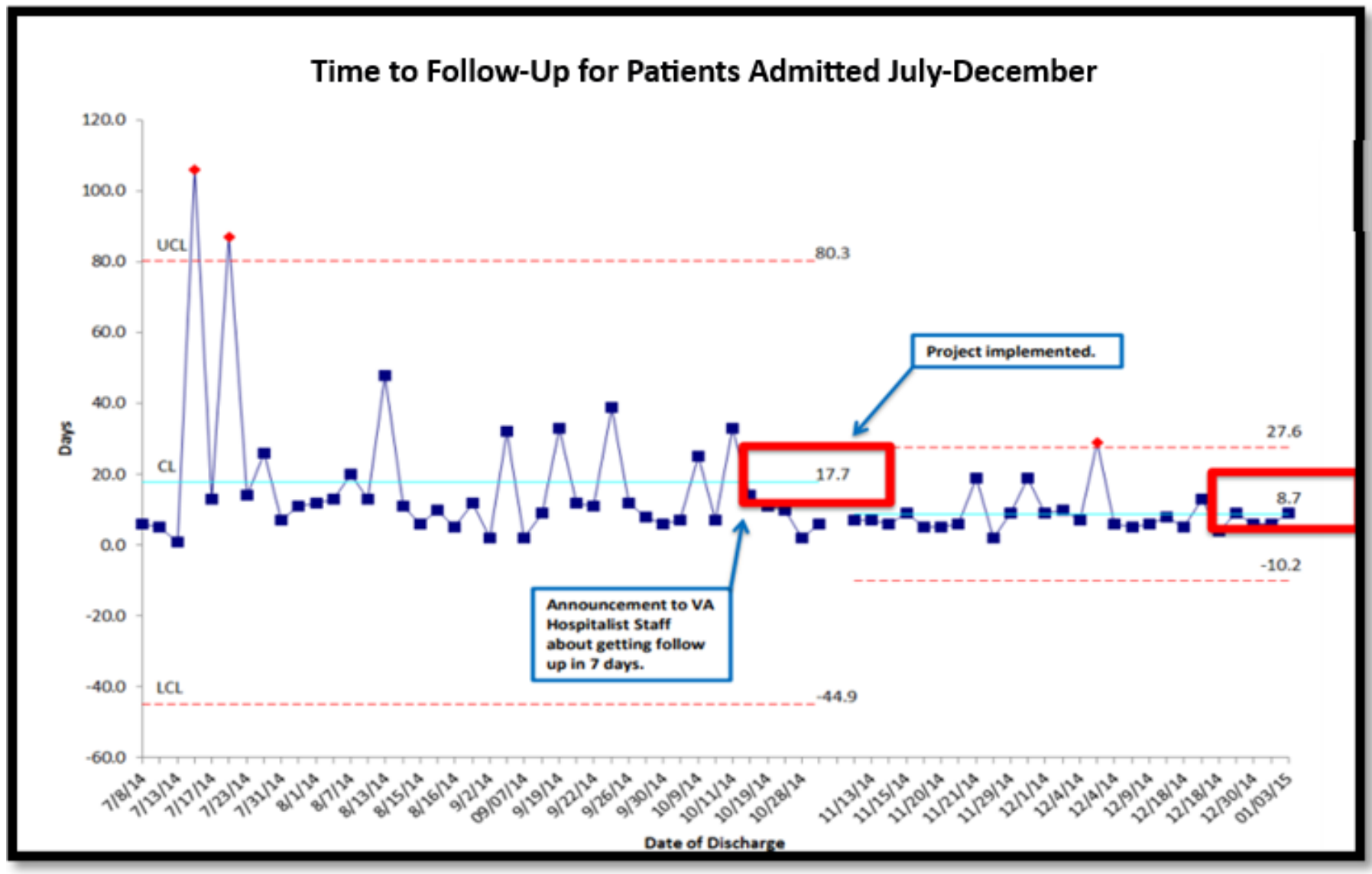

Figure 3 Control chart with split control limits before and after intervention. LCL, lower control limit; UCL, upper control limit. 
as death rate, complications and patient satisfaction, as well as overall efficiency and physician capacity at individual VA Medical Centers. ${ }^{9}$ SAIL uses the Centers for Medicare \& Medicaid Services' 30-day Congestive Heart Failure Risk-Standardized Mortality Rate to report the risk-adjusted 30-day mortality rates for $\mathrm{CHF}^{10}$ ).$^{910}$

We compiled this study in accordance with the Standards for Quality Improvement Reporting Excellence version 2.0 guidelines. ${ }^{11}$

\section{RESULTS}

Primary outcome measure follow-up completion rate

Our 7-day completed follow-up rate was 57\% (compared with $47 \%$ prior to this project). The difference in these percentages were not statistically significant, $\mathrm{p}=0.429$.

\section{Process measure time to follow-up}

Following the first intervention (education), we decreased the average time to follow-up from 17.6 days (SD 21.38 days) when the project was implemented to 8.7 days (SD 5.7 days) (figure 3 ). The difference in time to follow-up was statistically significant, $\mathrm{p}=0.016 ; 95 \% \mathrm{CI}$ $(-16.08$ to 1.76$)$.

\section{Secondary measure 30-day local readmission rates}

By 2015 and following the second intervention (intense follow-up intervention protocol), we decreased the percentage of patients readmitted within 30 days after discharge to $21 \%$ compared with the baseline of $25 \%$ in 2012.

\section{Secondary measure 30-day mortality}

The risk-adjusted 30-day mortality rate at baseline in May 2012 was $10 \%$ and by the end of the study period in December 2015 had fallen to $7.16 \%$.

\section{DISCUSSION}

The primary aim of this project was to increase the number of completed 7-day follow-up appointments to $100 \%$ for all patients discharged from the internal medicine service with the diagnosis of ADHF. We were unable to meet this objective; however, there was a significant reduction in the average time to follow-up.

Additionally, the system evaluation helped us to engage multiple stakeholders who were involved in this process and get a better understanding of the root causes of the problem related to early follow-up visit completion. This information was critical and informed more targeted and strategic interventions that helped us with our secondary aim to improve 30-day readmissions. Importantly, though reduction in 30-day mortality was not an aim of the project, it was an unexpected but important outcome of the work.

One of the tenets of $\mathrm{QI}$ is the understanding that there is a 'gap between what we know and what we do'. ${ }^{12}$ In the development of our project we realised that providers understood in a broad sense that short-term follow-up was important, but they were unaware of the evidence that recommended a specific time interval to follow-up and they did not have a standardised, efficient nor sustainable process to help them meet this objective.

For this project, the first intervention focused primarily on education, and thus helped to emphasise evidencebased practice recommendations. Though education is sometimes touted as a weak intervention, it gave context to the importance of the work moving forward. This was critical in creating the sense of urgency that is often needed to lead change. ${ }^{13}$

In our academic centre, the house staff rotate through the hospital on a staggered schedule that results in at least one resident physician leaving and another joining the team on a weekly basis. This made education and training on $\mathrm{ADHF}$ discharges quite challenging. The idea to use the EMR as a tool to solve the standardisation and sustainability problem emerged from the residents themselves. As front-line workers, they were the ideal agents to generate ideas for change.

Use of the EMR to help prevent heart failure readmissions is in itself not a novel approach. ${ }^{14}$ However, the risk stratification and intense follow-up pathway built into the EMR proved to be innovative and highly effective.

Furthermore, by creating a mandatory pathway for scheduling this follow-up within the standard discharge order set, we were able to ensure that the protocol was followed regardless of the resident physician's prior awareness of it.

It is noteworthy to point out that though other literature surrounding improving 30-day readmission suggests that early physician follow-up improves outcomes, ${ }^{6}$ patients in this study who did not follow-up with a cardiologist first (ie, low-risk patients), instead followed up with a clinical pharmacist who had been trained to evaluate patients with heart failure. It is unclear if the effect would have been more robust with physician providers. Due to the increased demand on clinic availability and because investigation of special cause variation in our data (see figure 3 figure 1 , red points represent special cause variation, and represent patients with extended time to follow-up) revealed that patients with extended time before follow-up with a provider were having nurse visits, we hypothesised that nursing follow-up alone did not result in changes to clinical management. This hypothesis was based on extensive chart review that revealed that the patients being seen by a nurse would have standing weights that were increasing (a marker for potential need for a titration in diuretic dose); however, no changes to diuretic dose were implemented, and no documentation of referral to a provider was made. Thus, the team decided to expand follow-up appointments to providers with training in physical exam skills pertinent to patients with CHF (which the PACT pharmacist received) and prescription writing authority (such as the PACT pharmacists). 


\section{LIMITATIONS}

There are several limitations to this study. First, we only evaluated patients with heart failure admitted to internal medicine services. The rationale behind this decision was that over two-thirds of ADHF admissions to our hospital go through internal medicine and unlike the cardiology service, these teams do not have dedicated nurse practitioner support assisting with follow-up scheduling. Newly diagnosed patients and patients with end-stage heart failure comprise most admissions to the cardiology service, and these sicker patients were excluded from our analyses.

Second, though readmission and mortality decreased, it is not possible to draw meaningful conclusions about the effect of specialist follow-up on patients we classified as high risk for readmission. The confounder is that the patients seen by a specialist still had early follow-up and it is unclear if the time to follow-up or the follow-up by the specialist provider was responsible for the improved outcomes.

Another important limitation has to do with the use of pharmacists instead of clinicians for evaluation of patients with heart failure. As physical examination is an essential part in diagnoses and triage of patients with heart failure and since this is not traditionally part of pharmacy training, there is ultimately some concern about accurate diagnosis, triage to home versus a higher level of care, and treatment in these patients. However, the pharmacists had access to clinic providers as needed.

Finally, though our intervention was successful at reducing readmissions, we did not achieve our primary aim to increase the number of completed 7-day follow-up appointments to $100 \%$.

We suspect that there are several potential patient, provider and system-level factors that prevented us from achieving our primary aim. First, even with the addition of pharmacists as providers, there may still have been a lack of provider availability to meet the needs of the discharged patients on such short notice. This was noted anecdotally when talking with the clerks in charge of scheduling. Another potential factor is a lack of appointment reminders. We know from prior literature ${ }^{15}$ that follow-up phone calls and reminders after discharge can improve compliance in making follow-up appointments. Lastly, for many of these patients, following up in general may present significant logistical challenges in terms of mobility, transportation or prioritising conflicting appointments with other providers.

These potential factors may be ameliorated using a dedicated heart failure 'navigator': a mid-level provider who can both remind patients to follow-up with an early postdischarge follow-up phone call and provide access to follow-up care. Additionally, a programme that would allow providers to make home visits could also be very beneficial. These interventions have been proposed and will be part of future studies.

Despite the limitations, this project does have several strengths. First, this was a resident-driven QI initiative and as representative stakeholders in the system, the residents were extremely effective at generating ideas for change. Second, the inclusion of residents into the planning and execution of the intervention required no extra financial support, and it fulfilled a requirement of their training by exposing them to key elements of QI. Finally, though resident physicians were heavily involved, this was a multidisciplinary effort with considerable involvement from nursing, pharmacy and clerical staff. The input of all these stakeholders was invaluable. Indeed, it is the combined efforts of the resident cohort, the Heart Failure Workgroup and the diverse multidisciplinary team members that led to improvement in this project.

\section{CONCLUSIONS}

How to reduce heart failure readmissions continues to be an area of intense national interest, but with marginal successes. ${ }^{4}$ In this paper, we describe a method for involving trainees at the point of care to both identify and apply evidence-based solutions to help reduce heart failure readmissions in our institution.

Our method was the result of iterative changes over multiple improvement cycles and resulted in a novel approach to solving a persistent problem. Our results demonstrate that risk stratification is a useful strategy to efficiently allocate limited resources to the patients with the most need. Furthermore, we found that using an EMR to enhance adherence to a protocol can also be an effective intervention. In patients with heart failure at our hospital, these strategies resulted in improved time to early follow-up, reduced 30-day readmission rates and decreased mortality.

Acknowledgements The authors acknowledge the following individuals and groups who helped make this publication possible: information technology expert, Patrick Gonzales; biostatistical support, George Mwinnyaa, MHS; internal medicine residents at the University of Texas Health San Antonio.

Contributors SM0 and JP contributed to the design and implementation of the research. SM0, JP, AG and JRD contributed to the analysis of the results and to the writing of the manuscript.

Funding The authors have not declared a specific grant for this research from any funding agency in the public, commercial or not-for-profit sectors.

Competing interests None declared.

Patient consent for publication Not required.

Ethics approval This project was deemed quality improvement by the Institutional Review Board at University of Texas Health San Antonio and received an exempt status.

Provenance and peer review Not commissioned; externally peer reviewed.

Open access This is an open access article distributed in accordance with the Creative Commons Attribution Non Commercial (CC BY-NC 4.0) license, which permits others to distribute, remix, adapt, build upon this work non-commercially, and license their derivative works on different terms, provided the original work is properly cited, appropriate credit is given, any changes made indicated, and the use is non-commercial. See: http://creativecommons.org/licenses/by-nc/4.0/.

\section{REFERENCES}

1. Desai AS, Stevenson LW. Rehospitalization for heart failure: predict or prevent? Circulation 2012;126:501-6. 
2. Munir MB, Sharbaugh MS, Thoma FW, et al. Trends in hospitalization for congestive heart failure, 1996-2009. Clin Cardiol 2017;40:109-19.

3. Benjamin EJ, Blaha MJ, Chiuve SE, et al. Heart disease and stroke statistics-2017 update: a report from the American Heart Association. Circulation 2017;135:e146-603.

4. O'Connor CM. High Heart Failure Readmission Rates. JACC Heart Fail 2017;5:393.

5. Centers for Medicare and Medicaid. Hospital compare on medicare. gov.

6. Hernandez AF, Greiner MA, Fonarow GC, et al. Relationship between early physician follow-up and 30-day readmission among medicare beneficiaries hospitalized for heart failure. JAMA 2010;303:1716.

7. Ryan J, Kang S, Dolacky S, et al. Change in readmissions and followup visits as part of a heart failure readmission quality improvement initiative. Am J Med 2013;126:989-94.

8. Avaldi VM, Lenzi J, Urbinati S, et al. Effect of cardiologist care on 6-month outcomes in patients discharged with heart failure: results from an observational study based on administrative data. BMJ Open 2017;7:e018243.

9. U.S. Department of Veterans Affairs Strategic Analytics for Improvement and Learning (SAIL). Value model measure definitions.
https://www.va.gov/QUALITYOFCARE/measure-up/SAIL_definitions. asp

10. Strategic Analytics for Improvement and Learning (SAIL). Value model measure definitions - quality of care.

11. Ogrinc G, Davies L, Goodman D, et al. SQUIRE 2.0 (Standards for QUality Improvement Reporting Excellence): revised publication guidelines from a detailed consensus process. BMJ Qual Saf 2016;25:986-92.

12. Grimshaw J, Eccles M, Thomas R, et al. Toward evidence-based quality improvement. 1966.

13. Kotter JP. Leading change:why transformation efforts fail by why transformation efforts fail. Business 2000;64.

14. Finkel N. Nine ways hospitals can use electronic health records to reduce readmissions. Hospitalist $2014 \mathrm{https}$ ://www.the-hospitalist. org/hospitalist/article/122869/9-ways-hospitals-can-useelectronic-health-records-reduce-readmissions (Accessed 28 Jan 2018).

15. Riegel B, Carlson B, Kopp Z, et al. Effect of a standardized nurse case-management telephone intervention on resource use in patients with chronic heart failure. Arch Intern Med 2002;162:705. 